states and position operators has much significance. Such doubts might arise particularly strongly if one is inclined to consider the collision matrix as the future form of the theory. One must not forget, however, that the customary exposition of this theory refers only to questions about cross sections. There is another interesting set of questions referring to the position of the scattered particles: how much further back (i.e., closer to the scattering center) are they than if they had gone straight to the scattering center and then continued in the new direction without any delay. ${ }^{15} \mathrm{In}$ order to answer such questions in the relativistic region, one will need some definition of localized states for elementary systems. From this point of view it is satisfactory that the localized states could be defined without ambiguity just for these systems.

${ }^{15}$ L. Eisenbud, Princeton Dissertation (1948).

\title{
On the Space-Time Geometry of a Moving Rigid Body
}

\author{
A. D. Fokker \\ Natuurkundig Laboratorium van Teyler's Stichting, Haarlem, Nederland
}

\begin{abstract}
A simple argument is given to explain Herglotz' theorem that a rigid body has three degrees of freedom only. A new example of rigid motion is given.
\end{abstract}

$\mathbf{I}_{\mathrm{a}}^{\mathrm{N}}$ $\mathrm{N}$ this paper I propose to consider in some detail another concept modified by relativity theory, the concept of a rigid body and its motions. In physics a rigid body is to be a collection of particles keeping rigid equal distances. In the theory of invariants a particle must not be represented by a point, but, being a lasting event, it is represented by a time track. Thus a rigid body must be taken to be a collection of time tracks continuously bearing constant distances between them.

The distance of two time tracks must be conceived as the interval along a straight line intersecting both tracks at right angles, in the sense of $(1+3)$-dimensional geometry. Thus at a certain instant of one time track the vectors showing the distances to neighboring tracks will lie in a space perpendicular to the time track, and they will continue to do so at following instants.

Now let $P Q R S T$ in Fig. 1 delimit successive infinitesimal segments of a time track. The time track can be seen as generated by successive infinitesimal "revolutions." (A "revolution" in (1+3)-dimensional timespace sometimes means an acceleration.) Take the

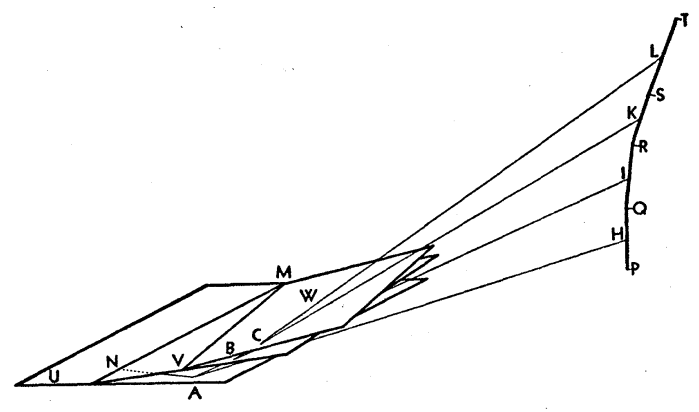

FIG. 1. spaces normal to the segments $P Q$ and $Q R$ respectively, bisecting these segments. The two spaces will intersect in a plane, $U$ say, which contains the points at equal distances from $P, Q$ and $R$. Therefore the arc $P Q R$ will be generated by an infinitesimal revolution (in $(1+3)$ dimensions) about the plane $U$. Again, the space normal to the segment $R S$ and bisecting it, will cut the bisecting space of $Q R$ in a plane $V$, and the arc $Q R S$ is generated by an infinitesimal revolution about the plane $V$. The straight intersection of $U$ and $V$ will contain the points equidistant from $P, Q, R$ and $S$. Finally the normal space bisecting the segment $S T$ will provide an intersection plane $W$ which is the "axis" of revolution generating the arc $R S T$. The intersection $M$ of the three $U, V$ and $W$ has equal distances from all five $P, Q, R, S, T$. It is the center of the osculatory equilateral superhyperboloid.

The osculatory plane containing $P Q$ and $Q R$ must be normal to the axis of revolution $U$. It is shown by $H I A$, where $A$ is the point of intersection with $U . H A$ is the principal normal of the time track, $A$ being the center of the flat hyperbole defined by $P, Q, R$. If we drop $A N$ perpendicular to $N M, N$ will be the centre of the one-blade hyperboloid defined by $P, Q, R, S$. This $A N$ is to be regarded as the binormal. Finally $N M$ is the trinormal of the time track $P Q R S T$.

When the series of infinitesimal revolutions about $U, V, W \cdots$ is generating the time track $P Q R S T \cdots$ any other points $P^{\prime}, P^{\prime \prime}$, situated in the same simultaneous space (in the same flat present, say), defined by $P$ and $U$, will generate time tracks $P^{\prime} Q^{\prime} R^{\prime} S^{\prime} T^{\prime} \cdots$, $P^{\prime \prime} Q^{\prime \prime} R^{\prime \prime} S^{\prime \prime} T^{\prime \prime} \ldots$. Two things are obvious. All these time tracks will always be normal to the flat presents $P, P^{\prime}, P^{\prime \prime} \cdots$ or $Q, Q^{\prime}, Q^{\prime \prime} \cdots$. The distances between 
two tracks will be given by vectors in these spaces, $P P^{\prime}$ and $P P^{\prime \prime} \cdots$, and they will be invariant during the generation of the tracks: $P P^{\prime}=Q Q^{\prime}=R R^{\prime}=S S^{\prime}$ $=T T^{\prime}=\cdots$.

Obviously the collection of time tracks generated in this way by a common succession of infinitesimal revolutions represents what must be called the motion of a rigid body.

The time tracks originating from points situated in another flat present space containing $U$ (not containing $P$ ), as $\pi \kappa \rho \sigma \tau \cdots$, generated by the same succession of infinitesimal revolutions about $U, V, W \cdots$ will show the motion of a different rigid body $\left(\pi, \pi^{\prime}, \pi^{\prime \prime} \ldots\right)$, related in a special way to the motion of the body $\left(P, P^{\prime}, P^{\prime \prime} \cdots\right)$.

In this way we see the motion of a rigid body as discussed by Herglotz ${ }^{1}$ and by Born. ${ }^{2}$ It is obvious that the time track of one particle, which alone is sufficient to determine the successive infinitesimal revolutions, determines all the time tracks. Therefore in relativity cinematics the rigid body has no more freedom than one single particle, three and only three degrees of freedom. It has no ordinary spatial rotation.

It is possible to choose, in the initial simultaneous present containing $U$ and $P$, an origin $O$ and three axes $O X, O Y, O Z$ defining a frame of reference, taking the track of $O$ for the axis of time. The motion of the rigid body carries this frame along, and we shall have an accelerated motion of a frame of reference free of rotation. If it is carried along a circular circumference, after completing a cycle, the axes will show a precession. This precession has become known as Thomas' precession. $^{3}$

Let us consider special cases. Instead of successive planes $U, V, W$, it might be that there is only one stationary "axis" of revolution, $U$ say. Take this to be the plane of $y, z$. The motions of the revolution will be defined by

$$
d t=x d \psi, \quad d x=t d \psi, \quad d y=d z=0
$$

Units have been chosen in order to reduce the velocity of light to unity. The resulting time tracks are equilateral hyperbolae and given by

$$
t=x_{0} \operatorname{Sh} \psi, \quad x=x_{0} \operatorname{Ch} \psi, \quad y=y_{0}, \quad z=z_{0} .
$$

The motions are hyperbolic accelerations. The acceleration for $\psi=0$ is given by

$$
a=1 / x_{0} .
$$

It is not the same in all parts of the rigid body. The acceleration tends to infinity near the plane of $Y$ - and $Z$-axes. The rigid body cannot extend beyond this plane into negative values of $x$.

In case the plane $U$ recedes to infinity, $x_{0} \rightarrow \infty$, this

${ }^{1}$ G. Herglotz, Ann. d. Physik 31, 393 (1910).

2 M. Born, Gött. Nachr. 1910, p. 161.

${ }^{3}$ L. H. Thomas, Nature 117, 514 (1926); Phil. Mag. 3, 1 (1927). Explicit integrated formulae are given in A. D. Fokker, Relativiteitstheorie (Noordhoff, Groningen, 1929) formula (12.25), p. 201. means that the hyperbolic time tracks straighten out the acceleration $a$ will vanish and the rigid body will show a stationary position or uniform rectilinear motion.

The case may be that during a revolution $d \psi$ about plane $U$, this plane shifts its orientation to $V$, the intersection of $U$ and $V$ being $M N$ and the angle $d \varphi$. Let this process be continued uniformly, with constant intersection $M N$ and a constant ratio $d \psi: d \varphi=\alpha: \beta$. Let $\alpha$ and $\beta$ be normalized, so that $\alpha^{2}-\beta^{2}=1$, and put $d \psi / \alpha=d \varphi / \beta=d s$.

Take four unit vectors $\tau, \xi, \eta, \zeta$ with $\tau^{2}=1, \xi^{2}=\eta^{2}=\zeta^{2}$ $=-1$, and $(\tau \cdot \xi)=(\tau \cdot \eta)=(\tau \cdot \zeta)=(\eta \cdot \zeta)=(\zeta \cdot \xi)=(\xi \cdot \eta)$ $=0$.

The vectors $\eta$ and $\zeta$ lie in the plane $U$, the vector $\zeta$ in the intersection of $U$ and $V$. The revolution $d \psi$ will carry $\tau$ and $\xi$ to new directions $\tau^{\prime}$ and $\xi^{\prime}$

$$
\tau^{\prime}=\tau+\xi d \psi, \quad \xi^{\prime}=\tau d \psi+\xi, \quad \eta^{\prime}=\eta, \quad \zeta^{\prime}=\zeta .
$$

The change of the axis of revolution $U$ into $V$ does not mean a motion, but the choice of new unit vectors $\xi^{\prime \prime}$ and $\eta^{\prime \prime}$ :

$$
\tau^{\prime \prime}=\tau^{\prime}, \quad \xi^{\prime \prime}=\xi^{\prime}-\eta^{\prime} d \varphi, \quad \eta^{\prime \prime}=\xi^{\prime} d \varphi+\eta^{\prime}, \quad \zeta^{\prime \prime}=\zeta^{\prime} .
$$

Adapting the vectors to the purpose of integration, one puts

$$
\tau_{a}=\alpha \tau-\beta \eta, \quad \xi_{a}=\xi, \quad \eta_{a}=-\beta \tau+\alpha \eta, \quad \zeta_{a}=\zeta .
$$

This leads to

$$
\tau_{a}^{\prime \prime}=\tau_{a}+\xi_{a} d s, \quad \xi_{a}^{\prime \prime}=\tau_{a} d s+\xi_{a}, \quad \eta_{a}^{\prime \prime}=\eta_{a}, \quad \zeta_{a}^{\prime \prime}=\zeta_{a}
$$

and consequently, after integration, to

$\tau_{a}=\tau_{0 a} C h s+\xi_{0 a} S h s, \xi_{a}=\tau_{0 a} S h s+\xi_{0 a} C h s, \eta_{a}=\eta_{0 a}, \zeta_{a}=\zeta_{0 a}$ or

$$
\begin{aligned}
& \tau_{a}=\tau_{0} \alpha C h s+\xi_{0} S h s-\eta_{0} \beta C h s \\
& \xi_{a}=\tau_{0} \alpha S h s+\xi_{0} C h s-\eta_{0} \beta S h s \quad \zeta_{a}=\zeta_{0} . \\
& \eta_{a}=-\tau_{0} \beta \quad+\eta_{0} \alpha
\end{aligned}
$$

Any point-instant is given by the end of a radius vector

$$
t_{0} \tau+r \cos \varphi_{0} \xi+r \sin \varphi_{0} \eta+z_{0} \zeta .
$$

By the revolution the unit vectors $\tau$ and $\xi$ change to $\tau^{\prime}$ and $\xi^{\prime}$; by substituting the plane $V$ for $U$ the coordinates $r \cos \varphi_{0}$ and $r \sin \varphi_{0}$ change to $r \cos \left(\varphi_{0}+d \varphi\right)$ and $r \sin \left(\varphi_{0}+d \varphi\right)$. The point-instant now is found at the end of the radius vector

$$
t_{0} \tau^{\prime \prime}+r \cos \left(\varphi_{0}+d \varphi\right) \xi^{\prime \prime}+r \sin \left(\varphi_{0}+d \varphi\right) \eta^{\prime \prime}+z_{0} \zeta^{\prime \prime} .
$$

It is clear that, using the adapted unit vectors $\tau_{a}, \xi_{a}$, $\eta_{a}, \zeta_{a}$ and integrating, the radius vector will be given by

$$
\tau_{a}\left\{\alpha t_{0}+\beta r \sin \varphi\right\}+\xi_{a} r \cos \varphi+\eta_{a}\left\{\beta t_{0}+\alpha r \sin \varphi\right\}+\zeta_{a} z_{0}
$$

where

$$
\varphi=\varphi_{0}+\beta s .
$$

Referring to the original unit vectors $\tau_{0}, \xi_{0}, \eta_{0}, \zeta_{0}$ one 
finds for the coordinates

$$
\begin{aligned}
& t=t_{0}\left(\alpha^{2} C h s-\beta^{2}\right)+r \cos \varphi \cdot \alpha S h s+r \sin \varphi \cdot \alpha \beta(C h s-1) \\
& x=t_{0} \cdot \alpha S h s+r \cos \varphi \cdot C h s+r \sin \varphi \cdot \beta S h s \\
& y=t_{0} \cdot \alpha \beta(1-C h s)-r \cos \varphi \cdot \beta S h s+r \sin \varphi \cdot\left(\alpha^{2}-\beta^{2} C h s\right) \\
& z=
\end{aligned}
$$

One verifies, that $t^{2}-x^{2}-y^{2}-z^{2}=t_{0}^{2}-r^{2}-z_{0}^{2}$, and that for $\beta=0$ the formulae reduce to those of the rectilinear hyperbolic acceleration found above.

Putting $t_{0}=0$ one finds the time tracks of a rigid body occupying the instantaneous present space at the origin of time. This motion has not been described by Herglotz.

Another conception of a rigid body has been put forward in early discussions, in which it was defined as a collection of points, not time tracks, preserving constant distances among them. The vectors connecting pairs of points which show the constant distance according to this conception need not be normal to the time tracks showing the motions of the points.

Any displacement of such a rigid body of the second kind is an example of a complex revolution in $(1+3)$ dimensional time-space, and in general the axis of such a complex revolution consists of a pair of planes, a 2-dimensional plane in space, and a $(1+1)$-dimensional plane in time-space (a separation plane and an inertia plane, to speak with Robb's terminology ${ }^{4}$ ) which are mutually perpendicular. A continued revolution about a separation plane means an accelerated motion. A revolution about an inertia plane is an instantaneous cyclic circular displacement.

Now it is obvious that one can conceive, as companions of the separation planes $U, V, W \cdots$ considered

${ }^{4}$ A. Robb, A Theory of Time and Space (1914); The Absolute Relations of Time and Space (Cambridge University Press, 1941). before, a series of inertia planes $U^{\prime}, V^{\prime}, W^{\prime} \cdots$, each perpendicular to its companion, every pair defined by a parameter $s$ and effectuating an acceleration or rotation $d \psi=f(s) d s$ and $d \chi=g(s) d s$ respectively. The succession of elements $U(s)$ and $U^{\prime}(s)$ would then generate a collection of time tracks or space curves permitting to locate, for every $s$, a collection of points preserving rigid distances. A few examples have been indicated by Herglotz. ${ }^{1}$

One may take a constant separation plane $U(=O Y Z)$ and a constant acceleration plane $(O X T)$ and make the uniform revolutions defined by $\psi=\alpha u$ and $\chi=\kappa u$. One will have the superposition of a rotation and an acceleration along the axis of rotation

$t=x_{0} S h \psi, x=x_{0} C h \psi, y=r \cos \chi, z=r \sin \chi, \psi / \alpha=\chi / \kappa=u$.

This kind of motions has been called by Herglotz the loxodromic group.

Again take a constant separation plane $U(=O Y Z)$ and a translation in it, the latter $(\lambda \psi)$ being the limit of a rotation about an acceleration plane $U^{\prime}$ at an infinite distance. The resulting group of motions is defined by

$$
t=x_{0} S h \psi, \quad x=x_{0} C h \psi, \quad y=y_{0}+\lambda \psi, \quad z=z_{0}
$$

and has been called by Herglotz the hyperbolic group.

Finally, one may take a stationary duration in time as the limit of an acceleration, and put it together with a circular displacement, $\chi=\omega t$. This leads to $t=t, x=x_{0}, y=r \cos \omega t, z=r \sin \omega t$. These circular motions were called by Herglotz the elliptic group. Obviously for $r \omega \rightarrow 1$ the velocity in the time tracks tends to the velocity of light. For larger $r$ one has no longer time tracks, but spatial curves, and so the particles of this rotating rigid of the second kind cannot extend beyond $r=\omega^{-1}$.

\title{
On the Motion of Test Particles in General Relativity
}

\author{
L. INFELd AND A. SCHILD* \\ University of Toronto, Canada
}

\section{INTRODUCTION}

$\mathbf{I}^{\mathrm{N}}$ this paper we give a simple derivation of the geodesic motion of test particles from Einstein's gravitational equations for empty space. The history of this problem is connected with the development of basic physical concepts.

Classical physics is dominated by a characteristic duality of field and matter. In Newton's theory of

\footnotetext{
*Now at Carnegie Institute of Technology, Pittsburgh,
}

gravitation as well as in the Maxwell-Lorentz theory of electromagnetism the physical laws fall naturally into two independent classes. The first class consists of the partial differential equations which (with suitable boundary conditions at infinity) determine the field in terms of the distribution and motion of the matter which "generates" it. The second class consists of the dynamical equations governing the motion of matter under the forces "exerted" by the field. The complete independence of the dynamical laws from the field 